\title{
The Effects of Anhedonia, Stress and Neurotic Personality on the Incidence of Depression
}

\author{
Xuemei Liang \\ The four affiliated hospital of Qiqihar Medical College; Heilongjiang Qiqihar 161000, China
}

Keywords: anxiety; stress and neuroticism; depression

\begin{abstract}
Patients with schizophrenia and depression have a reduced level of mental health, often with negative emotional growth and low self-evaluation. Self-pity is one of the objective criteria for mental health and is likely to be impaired. Anhedonia as a core clinical manifestation of schizophrenia and depression, but there is few research to explore the relationship between self-pity and anhedonia. There is no obvious difference in the symptoms of anhedonia between the two diseases, but there may be substantial differences in psychopathology. This difference is likely to have an impact on the association of self-pity and anhedonia. The purpose of this study is to understand the level of self-compassion in patients with schizophrenia and depression; to verify the relationship between self-pity and loss of anhedonia in patients with schizophrenia and depression; and to explore the effect of depressive symptoms on patients with both diseases and the different influences of the relationship between compassion and anhedonia.
\end{abstract}

\section{Introduction}

Anhedonia is the loss of one's ability to experience happiness. It is one of the core symptoms of depression. Clinically discovered anesthesia is difficult to cure. Even after a long-term high-dose antidepressant treatment does not significantly improve the symptoms, especially for patients with chronic depression. In a word, anhedonia may be a special attribute that will continue to affect the development of the course [1]. However, although anhedonia has an important role in the pathogenesis of depression, there is not much research on depression in terms of the specific emotional characteristics of anhedonia. Depression susceptibility theory, which centers on anhedonia, considers anhedonia as a special attribute of depression, has a genetic basis, and is prone to be stimulated by stress, and its mechanism is to reduce the discrimination of positive stimuli. Anhedonia affects the intensification process during childhood, that is, the response to negative stimuli increases, and the response to positive stimuli decreases. This process in turn affects the individual's cognitive style, so that the individual displays a large number of unpleasant experiences, accompanied by a reluctance The symptoms of introversion, likeness, pessimism, and negative compulsion, when the individual suffers from stress in adulthood, further aggravate its negative cognitive style, accumulating to a certain extent leading to depressive episodes [2]. Recently, Pizzagalli further proposed that stress and anhedonia lead to a neurobiological explanation of depression. He believes that anhedonia is caused by stress and imbalances in the brain's reward system. It finds that the susceptible individuals lose their dopamine secretion in the face of uncontrollable chronic stress and are blunted. The central dopamine neuronal pathway leads to the emergence of anhedonia and eventually leads to the development of depression under continuous stress [3]. It can be seen that anorgasmia is closely related to stress and plays an important role in the pathogenesis of depression. However, there is no correlation between anhedonia and stress in the pathogenesis of depression.

\section{Research Objects and Methods}

From May 2015 to July 2016, 168 patients with schizophrenia, 68 males and 104 females were selected for outpatient and inpatient departments at the Mental Health Center of Anhui Province. Age 18 to 50 (27.50 \pm 8.27$)$ years of age; 9 to 20 years of education (12.44 \pm 2.65$)$ years; Disease type: 
Paranoid 94 cases, undifferentiated 78 cases; Positive and Negative Symptom Scale score: Total score (46.33 \pm 13.96$)$ points, positive symptoms $(10.60 \pm 3.99)$ points, negative symptoms (12.73 \pm 6.62$)$ points; Beck depression scale scored $(7.06 \pm 8.30)$ points. The diagnosis meets the diagnostic criteria for schizophrenia in the 10th edition of the International Classification of Diseases (ICD-10) and was screened by the chief physician. (1) Enrollment criteria: 1 Diagnostic criteria for schizophrenia in line with ICD-10; 2 Ages 18 to 50; 3 Hans, right-handedness; 4 Junior high school or above, able to independently complete appropriate assessments; Voluntary participation in the experiment. (2) Exclusion Criteria 1 Conforms to ICD-10 Diagnostic Criteria for Other Mental Disorders; 2 has cerebral organic disease or other serious physical disease and has a history of severe traumatic brain injury; 3 has severe cardiovascular disease such as severe hypertension Diseases such as diabetes mellitus and hyperlipidemia; 4 have had substance dependence or abuse, such as alcohol, drugs, drug dependence, etc.; 5 electroconvulsive therapy has been performed in the past year.

From May 2015 to July 2016, using convenience sampling, a total of 112 depressive patients, 40 males and 72 females were selected in the outpatient and inpatient departments of the Anhui Mental Health Center. The age ranged from 18 to 49 (28.09 \pm 8.28$)$ years; the educational years ranged from 9 to $19(12.45 \pm 2.21)$ years; the Beck Depression Scale scored (13.63 \pm 6.89$)$ points. The diagnosis met the diagnostic criteria of ICD-10 depression and was screened by the chief physician. (1) The enrollment criteria 1 is consistent with the diagnostic criteria for ICD-10 depressive episode or recurrent depressive disorder; 2 ages 18 to 50; 3 Han ethnicity and above junior high school education; 4 right-handedness, able to cooperate with the completion of the corresponding assessment; 5 Voluntary participation in experiments and cooperation. (2) Exclusion Criteria 1 Conforms to ICD-10 Diagnostic Criteria for Other Mental Disorders; 2 has cerebral organic disease or other serious physical disease and has a history of severe traumatic brain injury; 3 has severe cardiovascular disease such as severe hypertension Diseases such as diabetes mellitus and hyperlipidemia; 4 have had substance dependence or abuse, such as alcohol, drugs, drug dependence, etc.; 5 electroconvulsive therapy has been performed in the past year.

From the affiliated hospital of the university and the community of the Anhui Mental Health Center, a total of 176 members were recruited, including 64 males and 112 females. Age 18 to 50 (27.37 \pm 9.98$)$ years of age; years of education 9 to $18(12.42 \pm 1.90)$ years (1) enrollment criteria 1 age, sex, education years matched the patient group; 2 ages 18 to 50; 3 Han ethnicity, right-handedness; 4 Junior high school or above, able to independently complete the corresponding assessment; 5 Voluntary participation in the experiment. (2) Exclusion criteria 1 Diagnostic criteria for all diseases that meet the ICD-10 mental disorders; 2 Have had an organic brain disease or other serious physical disease, have a history of severe brain trauma; 3 Have a history of substance dependence or abuse, such as alcohol, drugs , drug dependence, etc.

(1) name, sex, age, length of education, nationality, left/right handedness; (2) occupation, working years, marital status; (3) home address, contact information, and family contact information; (4) current Drug status, specific drug name and dosage; (5) family history of mental illness. The scale was used to measure the level of self-pity of the subjects. The scale is a self-assessment scale prepared by Neff in 2003 [4]. There are 26 items in total, divided into 3 subscales, namely self-friendliness, universal human nature and mindfulness. The indicators of self-pity level are the scores of the three subscales and the total scores of the three subscales. The scale is graded 1 to 5 . The higher the score, the higher the level of self-pity. The original scale has very good measurement properties with a test-retest reliability of 0.93 and internal consistency reliability of 0.92 . The confirmatory factor analysis can also be explained by a second-order factor. Domestic studies on university student populations have shown that the scale has good reliability and validity [5]. The alpha coefficients of this scale in the total sample, schizophrenia sample, depression sample and normal control sample are $0.859,0.847$, respectively. 0.864 and 0.836 .

The scale was compiled by Chapman et al. based on Meehl's theory and focused on assessing the degree of anodynamia in individuals from the perspective of social interpersonal communication. A total of 40 items were answered with "Yes" or "No". One point was scored consistently with the 
answer, which was a total score of 40 items. The higher the score, the worse the lack of social pleasure. A study of the Chinese general population and schizophrenia patients showed that the internal consistency reliability, split-half reliability, and test-retest reliability of the scale were 0.787 , 0.715, and 0.902, respectively. The scale was compiled by Chapman et al. based on Meehl's theory, and focused on assessing the degree of anodynamia of individuals from the aspect of physical sensation (such as eating and touching). It was divided into 61 items and answered with "Yes" or "No". A score of 1 was consistently scored, and the scale indicator was the sum of the scores of the 61 items. The higher the score, the more severe the loss of anorgasic body. A study of Chinese healthy people and schizophrenia patients showed that the internal consistency reliability, split-half reliability, and test-retest reliability of the scale were $0.850,0.796$, and 0.909 , respectively.

\section{Results}

There was no significant difference in gender, age, education, and positive events between the two groups. Depression group's anticipatory happiness, consumer happiness, and TEPS score were significantly lower than those in the healthy control group. Neurotic personality, negative events, stress scores, and BDI scores were significantly higher than those of the healthy control group. Sexual happiness, TEPS total score, neurotic personality, stress and BDI scores were correlated. The results showed that BDI score was significantly negatively correlated with expectant happiness, consumer happiness, and TEPS total score, as well as neurotic personality, negative events, and stress. The total score showed a significant positive correlation. Taking group as the dependent variable, the variables such as gender, age, education, expectation happiness, consumer happiness, TEPS total score, neurotic personality, BDI total score, positive events, and negative events were entered into the regression model, and the results showed that consumption Happiness, neuroticism, total BDI scores, and negative events have a significant predictive effect on the incidence of depression.

This study found that anhedonia is a core defect of depression. It has a significant effect on the occurrence of depression together with stress and neurotic personality. Compared with the normal control group, patients with depression have significant loss of expectation and consumer happiness. This is consistent with theoretical research. The loss of consumer pleasure is considered to be a more serious type of impairment and a sign of depression. The damage of the consumer happiness system is always accompanied by the damage of the expectant happy system. Neurotic personality scores, negative events, and stress scores were significantly higher in patients with depression than in healthy controls. This is consistent with the results of previous studies. Previous studies have shown that patients with depression have significant neurotic personality traits, more stressful life events, and individual depression symptoms may have a Heavy negative life events are related. In addition, correlation analysis found that the BDI score was significantly negatively correlated with expectant happiness, consumer happiness, and TEPS total score, indicating that anhedonia is closely related to depression, and the more severe anhedonia, the higher the degree of depression. Logistic regression analysis found that lack of consumer happiness, negative events, and neurotic personality were important factors in the pathogenesis of depression. Anhedonia, as a characteristic attribute of individuals, can easily lead to depressive episodes after experiencing a stressful situation during adolescence and adulthood. This study hypothesized that the loss of anhedrapies and the role of stress in the pathogenesis of depression may be based on a high neurotic personality, ie, a decrease in dopamine secretion caused by chronic stress, which induces the loss of anhedonia in neurotic personality individuals, and eventually under continuous stress. Depression is caused by the action. It is important that this study found that although there are two kinds of impaired expectancy and consumer happiness in patients with depression, only the lack of consumer happiness has entered the regression equation, and the expectant pleasure seems to have little effect on depression. The reason for the analysis may be that expectant happiness is related to trending motivation and is a trait, while consumer happiness is related to current emotions and is a state that is more severely damaged during the episode of depression. Klein also proposed that both expectant happiness and consumer happiness exist in depression at the same time, and the consumer happiness defect is 
more severe in the depressive episode.

\section{Conclusion}

The anticipatory happiness and consumer happiness of depression patients were significantly lower than those of healthy controls $(\mathrm{P}<0.05)$; neurotic personality, BDI, and stress were significantly higher than those of healthy controls $(\mathrm{P}<0.01)$; Logistic regression analysis found that consumer happiness $(\mathrm{OR}=1.10,95 \% \mathrm{CI}=1.03-1.17)$, neurotic personality (OR=0.91, 95\%CI=0.83-1.00), BDI (OR=0.93, 95\%CI=0.89-0.98), negative life events ( $\mathrm{OR}=0.94,95 \%$ $\mathrm{CI}=0.92-0.97)$ was significantly associated with the onset of depression. Conclusion: The individual's consumer happiness, negative stress and neurotic personality are risk factors for the onset of depression.

\section{References}

[1] Pyszczynski T, Greenberg J, Solomon S, et al. Why do people need self-esteem [J]? PsychologicalBulletin, 2004, 130 (3):435-468.

[2] Baumeister RF, Campbell JD, Krueger JI, et al. Does High Self-Esteem Cause Better Performance, Interpersonal Success, Happiness, or Healthier Lifestyles [J]? Psychological Science PublicInterest, 2003, 4(1):1-44.

[3] Crocker J, Park LE. The costly pursuit of self-esteem [J]. Psychological Bulletin, 2004, 130(3):392-414.

[4] Neff KD. The Role of Self-Compassion in Development: A Healthier Way to Relate to Oneself [J].Human Development, 2009, 52(4):211-214.

[5] Neff KD, Vonk R. Self-compassion versus global self-esteem: two different ways of relating tooneself [J]. Journal of Personality, 2009, 77(1):23-50. 